\title{
TRANSIENT MULTIPLE IMPULSIVE SIGNAL ANALYSIS FOR PNEUMATIC PERCUSSIVE RIVET TOOLS
}

\author{
John G. Cherng and Sheng-Lih Peng \\ Mechanical Engineering Department, University of Michigan-Dearborn, Dearborn, \\ Michigan 48128-1491, U.S.A.
}

(Received 20 March 1992, accepted 17 August 1992)

\begin{abstract}
This paper presents a systematic study on the analysis of the multiple impulsive transient signals generated by the pneumatic percussive rivet tools, i.e. rivet hammers and bucking bars. Detailed discussions are provided on how to conduct vibration measurements of the tools and the methods of analysing the multiple impulsive signals. Important issues such as triggering method, averaging, windowing, recording, and conversion from fast Fourier transform narrow band spectrum to a one-third octave-band spectrum are included in the paper. In addition, the implementation of ISO 5349 standard on this type of measurement is addressed. It is believed that the methods presented in this paper can be applied for similar transient signals generated by other types of mechanical systems.
\end{abstract}

\section{INTRODUCTION}

The pneumatic percussive rivet hammer and bucking bar are a set of tools that are generally used in aircraft and heavy vehicle constructions. However, the measuring and analysis of the vibration generated by these tools is not an easy task. The operation of the percussive rivet hammer is very different from the compression riveter or other pneumatic hand tools. The duration of the operation lasts less than $1 \mathrm{~s}$ with extreme high impulsive forces. These impulse forces create transient multiple impulsive signals which require special measurement setups on the analysers and analysing methods to obtain meaningful results.

The International Organization for Standardization published a guideline, ISO 5349 standard [1]. This ISO standard requires the measured vibration to be presented in a one-third octave or an octave frequency spectrum from $6.3 \mathrm{~Hz}$ to $1.25 \mathrm{kHz}$. This requirement adds another level of complexity in measuring the transient multiple impulsive signals that is not found in other hand tools' vibration measurements [2-4].

Research work that has been done in this field provides valuable information on some key issues but lack of details. Radwin and Armstrong [5] conducted an assessment study of the hand vibration exposure on an assembly line where the workers were using powered screwdrivers. A one-third octave acceleration spectrum was generated by using a fast Fourier transform (FFT) analyser while the tool was operated continuously on a specially designed fixture. Their study suggested a solution on how to overcome the problem of short operation time of a pneumatic tool. However, a FFT analyser and continuous operation do not automatically produce a correct one-third octave frequency spectrum. O'Connor and Lindquist [6] have discussed the non-linear effects of accelerometers leading to DC shifts in measuring the vibration of a chipping hammer. They suggested that a low-pass mechanical filter should be used with a regular piezoelectric accelerometer instead of a 
shock accelerometer. Their study confirmed the recommendation made by the ISO standard that a low-pass mechanical filter is recommended in performing vibration measurements of pneumatic percussive tools. A back-to-back method was used to compare the readings of two accelerometers. Unfortunately, no information was given on the analyser setup and the measuring method. Engstrom and Dandanell [7] presented a study on the riveting tools based on the ISO standard. They had experienced the difficulty on complying with the ISO standard to generate the weighted one-third octave acceleration spectrum. They did not give solutions to this problem in the paper. Instead, they suggested that a complement to the ISO standard is needed for the measuring method and analysing technique of the percussive tools.

It was noticed that almost all the studies [2-7] included the one-third octave spectrum as required by the ISO standard but none of them had shown how this one-third octave band spectrum could be calculated. We have found that the calculation of a one-third octave frequency spectrum of these percussive tools is not as straight forward as it appears to be. It was also noticed that engineers still use the conventional measuring method with a digital analyser without looking into the limitations of the analyser.

The objectives of this paper are twofold: (1) to review the key issues related to the transient multiple impulsive vibration measurement and (2) to present the measuring methods and analysing techniques that will generate correct results and will also comply with ISO standard.

\section{MEASURING THE TRANSIENT MULTIPLE IMPULSIVE VIBRATION}

Figure 1 shows a typical time signal recorded from a rivet hammer. The duration of the hammering is approximately $350 \mathrm{~ms}$ with a total of nine hits to flatten a $3.175 \mathrm{~mm}$ dia. rivet. The time difference between two hits, i.e. two peaks, is approximately $40 \mathrm{~ms}$. The fundamental hitting frequency will be about $25 \mathrm{~Hz}$ or $1500 \mathrm{blows} / \mathrm{min}$. The impulsive force is first in the negative direction then immediately changes to the positive direction. The time signal from a bucking bar is similar to a rivet hammer except that the impulsive force is first in the positive direction and then changes to the negative direction.

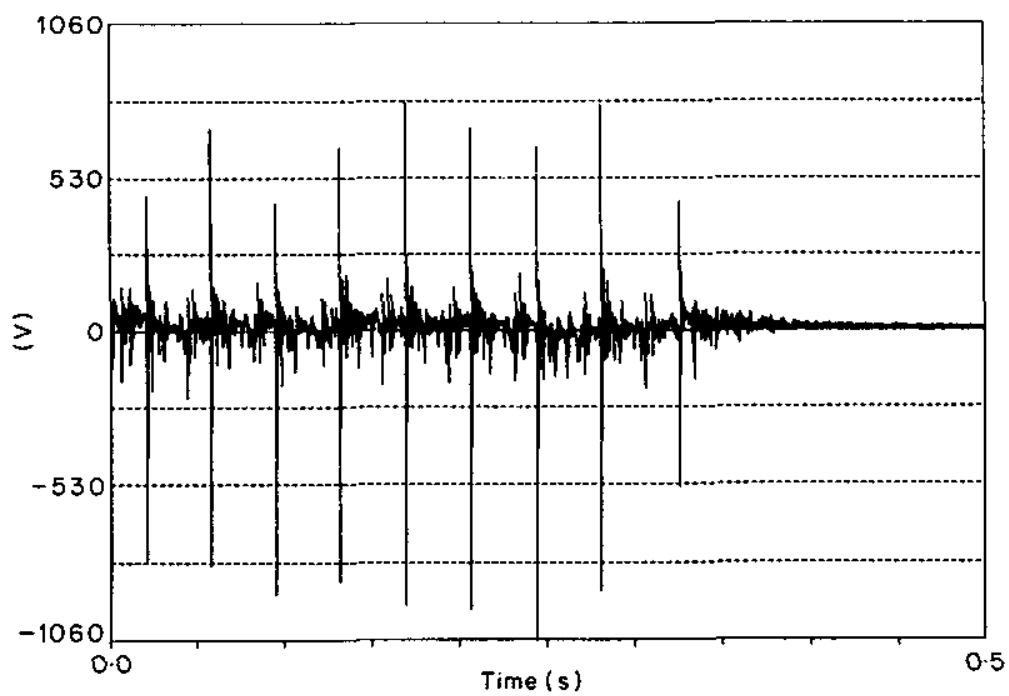

Figure 1. Typical time signal for a rivet hammer. 
The ISO standard requires that the measured vibration be presented in a one-third octave band with center frequencies from $6.3 \mathrm{~Hz}$ to $1.25 \mathrm{kHz}$. The one-third octave band frequency analysis calls for a continuous or steady-state vibration measurement. Difficulties were experienced by other researchers [7] in applying the ISO standard to the measurement of transient multiple impulsive vibration generated by percussive rivet tools. This section will discuss the relevant measurement and display setups of the two most commonly used analysers, i.e. the real time digital filter analyser (DFA), and a FFT analyser. Additionally, the difficulties encountered in meeting the ISO standard are discussed.

\subsection{KEY ELEMENTS OF VIBRATION MEASUREMENT}

The key elements in measuring the transient impulsive signals consist of a mechanical filter, an accelerometer, a charge amplifier, an analyser, a computer and a printer/plotter.

The input signals, i.e. accelerations, can be sensed by an accelerometer which should have a resonant frequency above $25 \mathrm{kHz}$ as required by the ISO 5349 standard. The accelerometer should be calibrated before and after each test according to the manufacturer's specifications. A low-pass mechanical filter is installed between the vibration surface and the accelerometer. The mechanical filter and the accelerometer should be tightly connected by adding a threaded stud between them. The mechanical filter can also be threaded into the surface of the handle of the rivet hammer or by other suitable means.

A charge amplifier with low-pass filter is preferred, such as the B \& K 2635 which has an adjustable low pass filter ranging from $100 \mathrm{~Hz}$ to $100 \mathrm{kHz}$. The cut-off frequency should be set at $3.0 \mathrm{kHz}$ as required by the ISO 5349 standard.

As the ISO standard requires the measured acceleration to be presented in an octave or one-third octave frequency band, it appears that a real time octave band DFA is the natural selection. On the other hand, an FFT analyser is commonly used for vibration measurements. However, both types of analysers have their shortcomings in measuring transient multiple impulsive signals generated from percussive rivet tools. More detailed discussions on this issue are presented later in this section.

A personal computer is generally connected to the digital analyser through an IEEE Board. The personal computer has many advantages for signal analysis such storing and retrieval of the acquired data, conversion of a FFT spectrum to a one-third octave band spectrum and calculating the weighted acceleration spectrum.

A printer and/or a plotter is interfaced with the analyser and the computer to print and plot the frequency spectrum and the calculated results.

\subsection{RELEVANT ANALYSER MEASUREMENT AND DISPLAY SET-UP}

The measurement functions and display of an analyser should be set properly. The characteristics of the rivet tools' signals require that special attention is paid to the analyser settings, these are generally overlooked by researchers and engineers. The following discussions are based on using a B \& K 2034 dual channel FFT analyser and a B \& K 2133 dual channel digital filter analyser.

\subsubsection{Trigger method}

The external trigger method should be used to catch the extreme front end of the impulsive signal. A free run method should not be used as the signal is shorter than a record length. Data sampling can be triggered either by a positive or a negative slope depending on which matches the direction of the signal. The level of triggering can be either a positive or negative percentage of the maximum value of the vibration. In our experience, a positive slope with a level at -10 per cent is a reasonable value to use for the rivet 
hammer and the negative slope is better for a bucking bar. Delay triggering may also be used to ensure a good match with the input signal. After a few trials and errors, one should be able to get a good feeling about the signal characteristics and thus select a proper value.

\subsubsection{Number of averages}

Since the duration of the signals is less than a record length, one linear average is recommended for a FFT analyser and a single input is recommended for a real time DFA.

\subsubsection{FFT weighting window}

A linear or rectangular window is recommended for a FFT analyser. All parts of the signal are equally weighted in a rectangular window. In the frequency domain, the bandwidth of the signal is greater than the bandwidth of the filters, because the signal is shorter than the record length, therefore the filter characteristic will have no influence on the calculated spectrum of the transient signal. A Hanning window is inappropriate because the signal is not continuous during the recording time. An exponential window cannot be used because the signal is not longer than the record length. A transient window should be used after the signal is recorded and the length of the window can be properly adjusted. This is discussed in the next section.

\subsubsection{Recording}

A single recording should be used to match the single average other than the continuous recording. The analyser will record one triggered signal and stop immediately.

\subsubsection{Anti-aliasing filter setting and $A C$-coupling}

The anti-aliasing filter should be selected at the maximum frequency range, e.g. $25 \cdot 6 \mathrm{kHz}$ for a B \& K 2034, to take advantage of the high sampling frequency of the analyser. The $3 \mathrm{~Hz}$ AC-coupling ( $3 \mathrm{~Hz} \mathrm{ACC}$ ) is also recommended if the analyser has this function in order to reduce DC errors.

\subsubsection{Display set-up}

The analyser display should be set at the time trace of its real value in linear co-ordinates. Using this display, one can check the time signal immediately after it is recorded. If the signal is not properly recorded, the problem should be identified and the test repeated. The frequency spectrum display should not be used during the data recording as one cannot tell the quality of the signal in this type of measurement.

\subsection{GENERATION OF THE FREQUENCY SPECTRUM}

The frequency spectrum from a time signal in a digital analyser is generally generated by a menu pick or a button push. Three fundamental factors that should receive special attention to generate the frequency spectrum of transient multiple impulsive signals: (1) the signal sampling frequency; (2) the signal sampling time, (3) the frequency resolution.

The sampling frequency of a digital analyser is derived from the Shannon or Nyquist theorem [8]:

$$
F_{s}=2 \times F_{\max }
$$

where $F_{\mathrm{s}}=$ sampling frequency and $F_{\max }=$ maximum frequency range.

The theorem states that a sampled time signal must not contain components at a frequency above half the sampling rate, i.e. the sampling rate has to be greater than or equal to two times the maximum signal frequency. However, in order to avoid the aliasing error, the sampling frequency is generally selected at a value larger than 2 . The most 
commonly used value is 2.56 . For most commercial analysers, the sampling frequency is automatically set respective to the maximum frequency range of the analyser. Thus, if an analyser has a $25.6 \mathrm{kHz}$ maximum frequency range, the sampling frequency will be about $65 \mathrm{kHz}$. This is much greater than the hitting speed of a rivet hammer and the required maximum frequency range of the ISO standard. Therefore, the sampling frequency of an analyser is not generally a problem in measuring the vibration generated from percussive rivet tools.

The requirement for the sampling time is based on a formula derived from an analog analyser, but it is also applicable to a digital analyser. The formula is the so called ' $B T$ product' which is defined as the product of the bandwidth and the sampling time of a given filter:

$$
B \times T \geqslant 1 \cdot 0
$$

where $B=$ bandwidth (Hz) and $T=$ sampling time (s).

Equation (2) implies that the product of the bandwidth of a given filter (or a display line on the screen of a digital analyser) and the sampling time has to be greater than or equal to $1 \cdot 0$.

In a real time DFA, an octave band digital analyser, the bandwidth changes with the centre frequency. At the low centre frequency range, the bandwidth becomes very small e.g. $2 \cdot 32 \mathrm{~Hz}$ for a $10 \mathrm{~Hz}$ centre frequency, $1.77 \mathrm{~Hz}$ for a $8 \mathrm{~Hz}$ centre frequency and $1.47 \mathrm{~Hz}$ for a $6.3 \mathrm{~Hz}$ centre frequency etc. Then, the required sampling time will be $431 \mathrm{~ms}$ for $2.32 \mathrm{~Hz}, 565 \mathrm{~ms}$ for $1.77 \mathrm{~Hz}$, and $680 \mathrm{~ms}$ for $1.47 \mathrm{~Hz}$ in order to meet the $B T$ product requirement. However, the operation time of a rivet hammer is less than $350 \mathrm{~ms}$. Therefore if a real time DFA is used to measure the transient multiple impulsive signals of the rivet tools there will generally be low confidence levels in low frequency bands.

Figure 2 presents a series of one-third octave band plots generated by a B \& K 2133 dual channel real time DFA. The rivet hammer was hitting against a metal bar. Four tests were performed with different operation times equal to $0.5,2 \cdot 0,4.0$ and $6.6 \mathrm{~s}$. It can be seen that accelerations in the low frequency range below $10 \mathrm{~Hz}$ gradually appeared on the spectrum as the sampling time increased. The confidence level which is indicated by a heavy line underneath the spectrum has been shortened from 125 to $10 \mathrm{~Hz}$ respectively. The heavy line indicates where the confidence reaches to within 68 per cent of a preset accuracy. The confidence level is calculated by the expected error which is determined by the following equation

$$
\text { expected error }=\frac{1}{2 \times \sqrt{B T}} .
$$

Equation (5) implies that the larger is the $B T$ product, the smaller are the expected errors or the higher the confidence level. When the duration of the transient signal is very short, the octave band DFA will have difficulties in obtaining high confidence levels in its low centre frequency bands.

A FFT analyser also has to meet the $B T$ product requirement, but the bandwidth of each frequency line of a FFT analyser is a constant. For example, a 800 spectral line FFT analyser for the frequency range $1.6 \mathrm{kHz}$ will have a bandwidth of $2 \mathrm{~Hz}$ for each spectral line. The required sampling time will be $500 \mathrm{~ms}$ which is slightly longer than the $350 \mathrm{~ms}$ of a rivet hammer. A post-transient window technique can be applied to a FFT analyser to provide an accurate frequency spectrum.

Figure 3 presents a post-transient window applied to a retrieved time signal generated by a rivet hammer [9]. The measurement and display setups of a B \& K 2034 FFT dual channel analyser discussed in the previous section are also shown in the figure. The length 


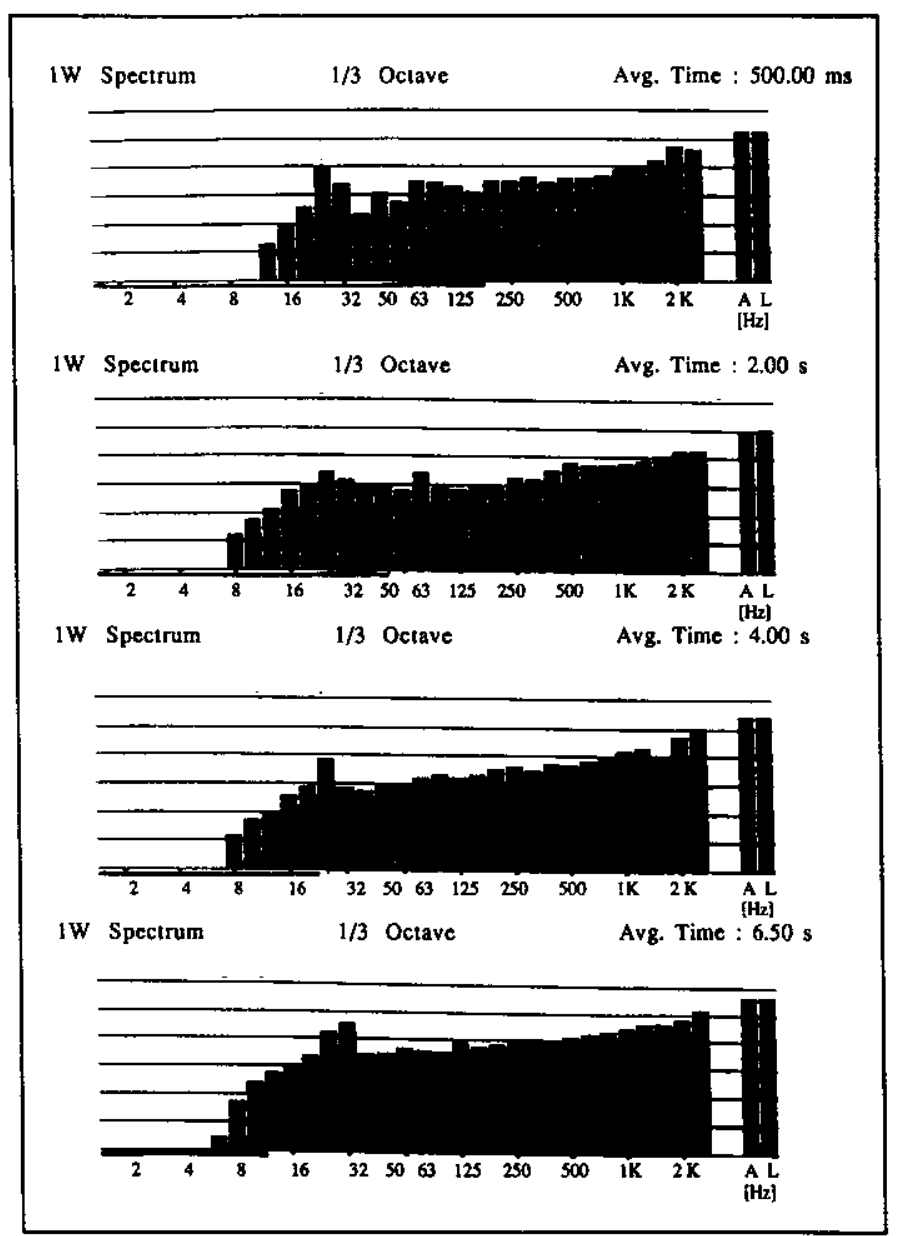

Figure 2. Confidence levels of one-third octave frequency spectra for sampling time at $0 \cdot 5,2 \cdot 0,4 \cdot 0$ and $6 \cdot 5 \mathrm{~s}$.

of the window covers all impulse signals other than the noises in the leading and trailing edges. Figure 4 presents the instantaneous spectrum of the time signal from the transient window. The low and high frequency harmonics of the signal are shown distinctively in the spectrum. This post-transient window technique can be applied to any transient impulsive signals generated from other types of mechanical systems to obtain a correct frequency spectrum.

The third issue regarding signal analysis is the frequency resolution of the analyser. The screen frequency resolution of a real time DFA is not an issue because the ISO standard only requires a one-third octave spectrum not a one-twelfth octave band. The commonly used real time DFA will have both octave and one-third octave spectra. Some of them may have a one-twelfth octave band.

FFT analysers generally have two types of screen resolution; 400 lines and 800 lines. Unfortunately, neither of them is sufficient for converting a $1.6 \mathrm{kHz}$ FFT spectrum into a $1.6 \mathrm{kHz}$ one-third octave frequency spectrum.

The equation to convert a FFT spectrum into a one-third octave spectrum is:

$$
A_{\mathrm{oct}, j}=\sum_{i=1}^{N}\left(A_{\mathrm{FFT}, i}\right)
$$




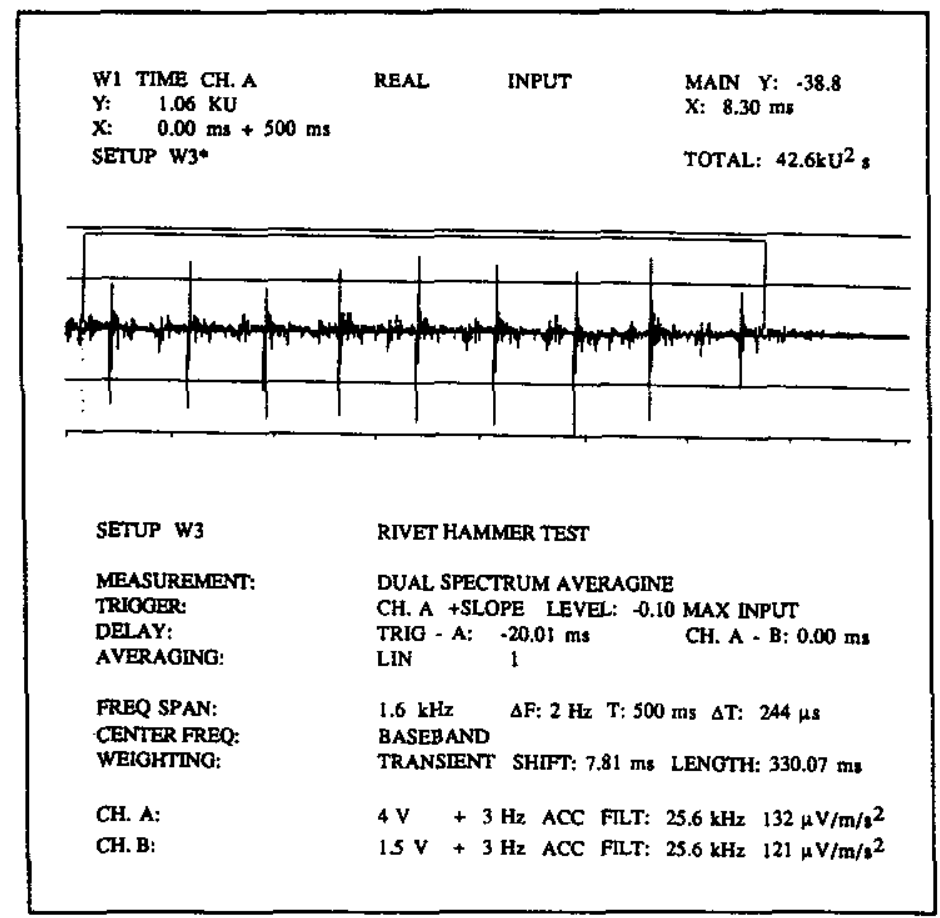

Figure 3. Post-transient window applied to a recorded time signal of a rivet hammer.

where: $A_{\text {oct }, j}$ is the acceleration at a one-third octave centre frequency $j ; A_{\mathrm{FFT}, i}$ is the FFT accelerations within the bandwidth of the centre frequency of a one-third octave band, $N$ is the total number of FFT spectral lines within the one-third octave bandwidth.

The value of $N$ should be at least 5 within the bandwidth of each centre frequency of the one-third band. However, in the low centre frequencies of a one-third octave band

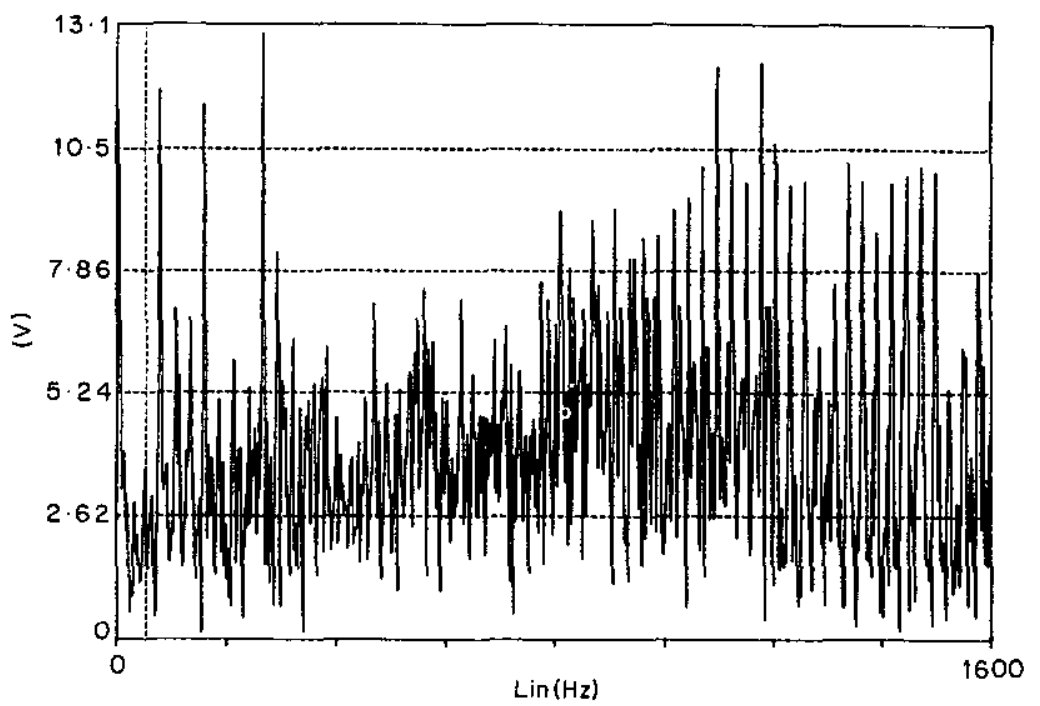

Figure 4. Instantaneous spectrum of the time signal captured by using a post-transient window. 
spectrum, the bandwidth is so small e.g. less than $4 \mathrm{~Hz}$ for the $16 \mathrm{~Hz}$ centre frequency, it will have difficulty in including enough readings from the FFT spectrum if the resolution of the spectrum is too large e.g. $2 \mathrm{~Hz}$ for each spectral line.

Digital analysers and conversion software usually truncate the spectral lines which do not have enough readings during the conversion process. In most cases, this truncation does not create significant errors because the energy in the low frequency band is very small in comparison with the energy in the rest of spectrum such as in noise measurements. However, the fundamental frequency of most percussive tools ranges from 25 to $40 \mathrm{~Hz}$. A large amount of energy can be within the low frequency bands. In addition, omitting the vibration in the low frequency bands will not meet the requirement of the ISO standard which requires a complete spectrum from $6.3 \mathrm{~Hz}$ to $1.25 \mathrm{kHz}$. The resolution of a FFT analyser is an important factor in human health-related vibration measurements.

\section{MEASURING METHODS FOR RIVET TOOLS}

We may conclude that both real time DFA and FFT analysers have their shortcomings in generating an ISO standard required one-third octave frequency spectrum for rivet tools. The real time DFA will have low confidence levels up to $125 \mathrm{~Hz}$ if the sampling time is less than $500 \mathrm{~ms}$. Although the FFT analyser can produce an accurate narrow band frequency spectrum by using the post-transient window technique, it has a resolution problem in converting a FFT spectrum to a one-third octave band spectrum. Different measuring methods and analysing techniques that can comply with the requirements of ISO standard are presented in this section. It is believed that these methods can be applied to similar transient signals generated by other types of mechanical systems.

\subsection{INTEGRATION OF TRANSIENT IMPULSE SIGNALS}

One method is to integrate many short transient impulse signals into a long series of impulse signals. The key element of the measurement is a tape recorder. An analog tape recorder is preferred, but a high sampling frequency $(>5 \mathrm{kHz})$ with a large dynamic range ( $>85 \mathrm{~dB}$ ) digital tape recorder would be adequate. The vibration signal output from the charge amplifier are recorded on the tape recorder. The recorded data is then played back to a real time DFA or a FFT analyser.

The measurement set-up of the analyser is similar to the previous set-up other than larger number averages with continuous recording should be used since the signal is continuously fed into the analyser. In addition, the Hanning window may be used in the FFT analyser since the signal is now longer than the record length. An external trigger may be set at a higher level e.g. 40 to 50 per cent of the maximum value so that it will not be triggered by signals that are not generated by the rivet hammer or the bucking bar. The display set-up of the analyser can be changed to auto spectrum so as to see the counts of the linear averages in a B \& K 2034 FFT analyser. It is good practice to occasionally check the signal in the time domain. Other analysers may see the number of average counts in the time domain and this is better.

Although the averaged signal may consist of signals from 50 rivetings they may not all be identical, the overall averaged signal is more statistically representative of the actual field operation than a single measurement. In actual operation, each riveting does vary from time to time. Certainly, from the statistical point of view, the result from a larger number of signals will be more representative than the result from a few signals.

If a real time DFA is used, the one-third octave band frequency spectrum can be generated directly. Since the duration of the integrated signal is longer than several seconds, the value of the BT product will be much greater than 1 . The spectrum will have 

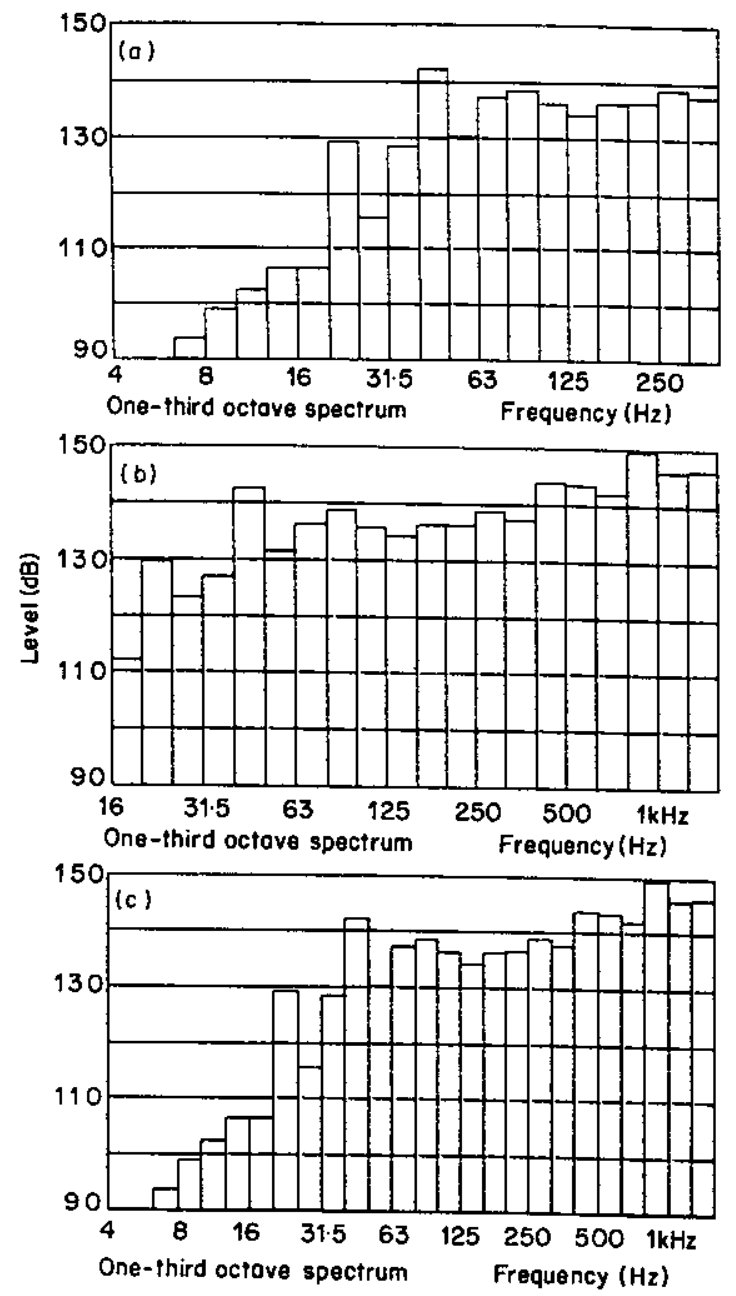

Figure 5. One-third octave band spectra for the frequency ranges (a) $4-400 \mathrm{~Hz}$, (b) $16-1.25 \mathrm{kHz}$ and (c) $4-1.25 \mathrm{kHz}$.

high confidence levels in both high and low frequency bands. This is the simplest method to generate a one-third octave frequency spectrum.

If the characteristics of the vibration are the main interest, e.g. the harmonics of the fundamental frequency, a FFT analyser should be used. A high frequency resolution FFT analyser such as one with 3200 display lines is desirable. However, one can always retrieve the data twice to a low resolution analyser at two different frequency range settings, such as one at $400 \mathrm{~Hz}$ and one at $1.6 \mathrm{kHz}$ for a 800 line FFT analyser. The data recorded in the $400 \mathrm{~Hz}$ frequency range will be in the $0.5 \mathrm{~Hz}$ bandwidth and can be used for the low frequency bands of a one-third octave spectrum. The data recorded in the $1.6 \mathrm{kHz}$ frequency range will be in the $2 \mathrm{~Hz}$ bandwidth, they can be used for the high frequency bands of a one-third octave spectrum. The two one-third octave band data are then integrated into a complete one-third octave band spectrum. Figure 5 shows three, two individual one-third octave band spectra from 4 to $400 \mathrm{~Hz}$ and from $16 \mathrm{~Hz}$ to $1.25 \mathrm{kHz}$, and the integrated spectrum from $4 \mathrm{~Hz}$ to $1.25 \mathrm{kHz}$. 


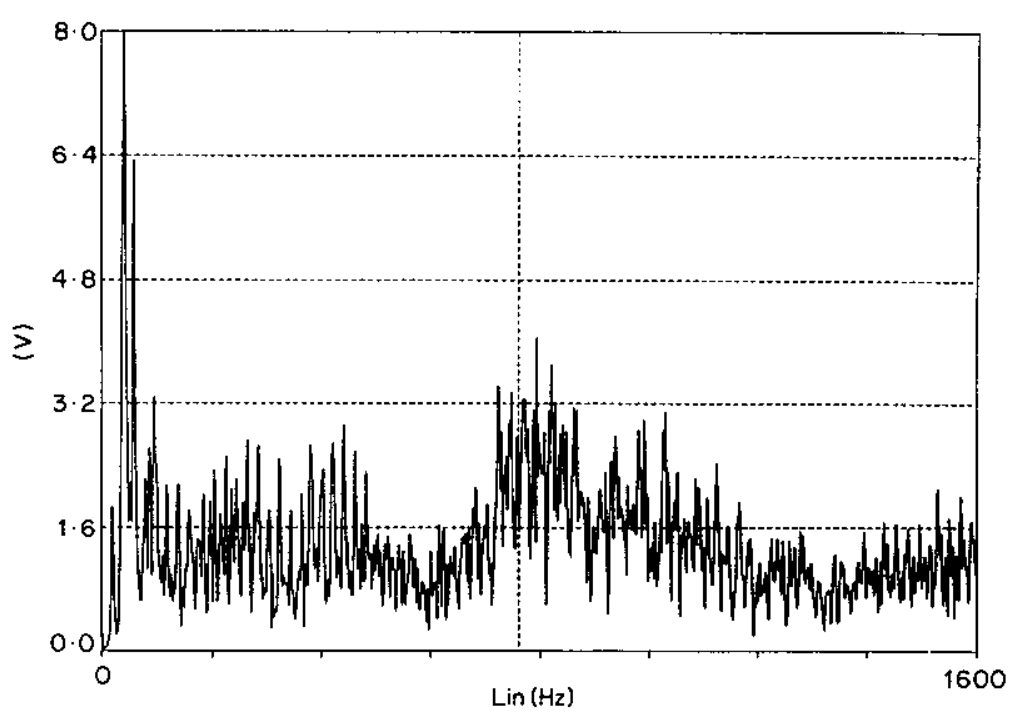

Figure 6 . Frequency spectrum generated by using the post-transient window method of a single time signal.

A case study was conducted to compare the post-transient window method and the integration of the transient multiple signal method. A rivet hammer was mounted on a test fixture. The vibration data from up to 50 runs were recorded onto a TEAC analog tape recorder. Figure 6 presents the spectrum generated by using only one signal from the tape with the post-transient window technique. The overall $\mathrm{rms}$ acceleration is $36.6 \mathrm{~m} / \mathrm{s}^{2}$. The harmonics of the vibration are very distinctive. Figure 7 shows the auto spectrum that was generated by 50 linear averages of the recorded data. The overall rms acceleration is $36.1 \mathrm{~m} / \mathrm{s}^{2}$. The harmonics of the vibration are not so distinctive in the higher frequency range. The broader responses may be due to the leakage errors that are generated by non-periodic input signals. However, the overall accelerations of the two measurements are very close. The integration method should be applicable to the vibration measurement.

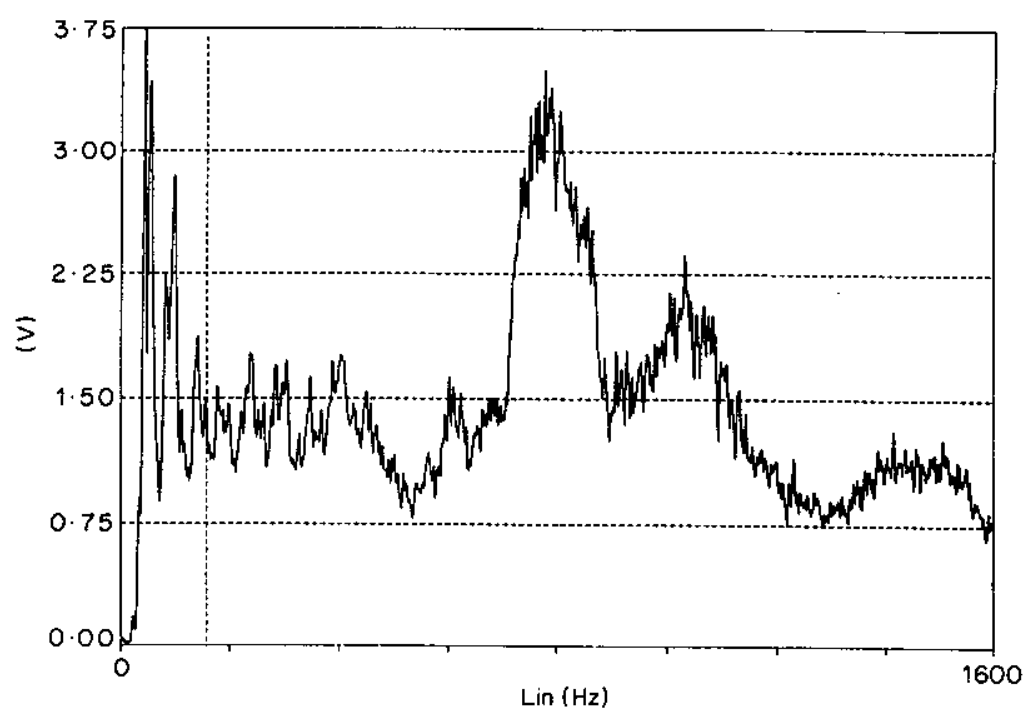

Figure 7. Frequency spectrum generated by 50 linear averages. 


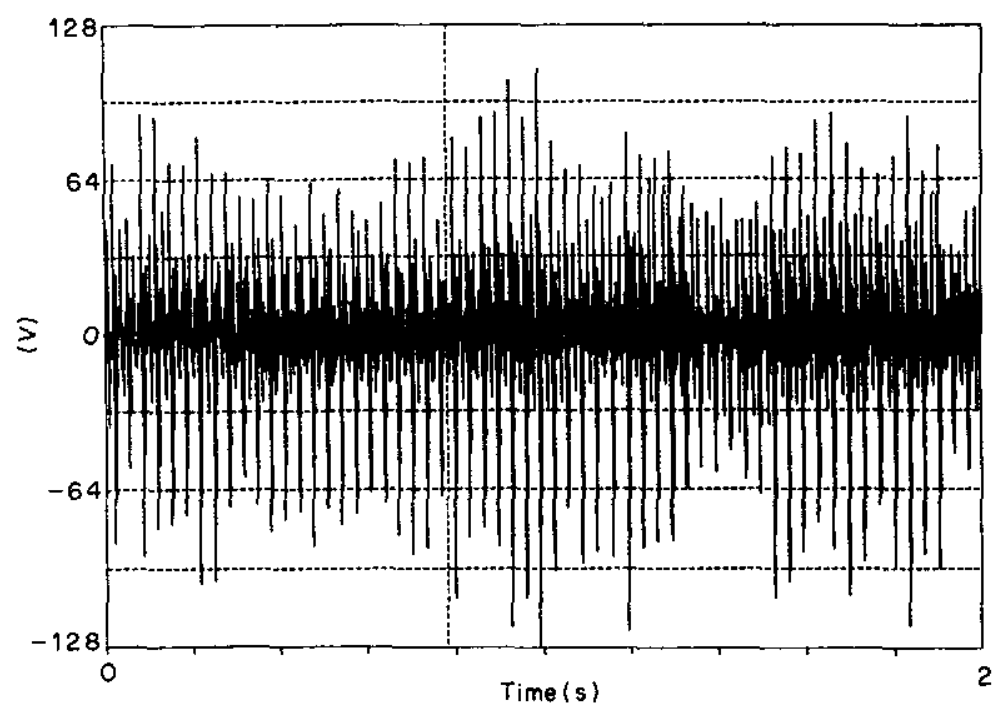

Figure 8. Time signal of a rivet hammer for a complete $2 \mathrm{~s}$ sampling time.

\subsection{EXTENDING THE OPERATION TIME}

The second method is to extend the operation time of the rivet tools. To operate the rivet hammer against a large metal bar or to mount the rivet hammer and/or the bucking bar on a test fixture extends the operation time of the rivet tools to several seconds or even longer.

Tool manufacturers generally test the rivet hammer against a piece of metal block. A flat surface ended rivet set is used instead of a regular bucket head rivet set. A worker presses down the trigger of the rivet hammer for a much longer period of time than usual, e.g. 5 to $10 \mathrm{~s}$, the rivet hammer continuously hits the metal block as long as the trigger is pressed down.

The advantages of using a human to conduct the testing are: (1) it is simple, (2) the rivet hammer is held in the same condition as when it operates in the field, and (3) more than one direction of vibration can be measured.

Figures 7 and 8 present a $2 \mathrm{~s}$ recorded time signal and its $400 \mathrm{~Hz}$ frequency spectrum respectively. The time signal fulfilled the complete sampling time and the harmonics are very distinctive in the spectrum. The bandwidth of each displayed line is $0.5 \mathrm{~Hz}$ on a 800 line resolution screen. This high resolution spectral information can be used to calculate the low frequency bands of a one-third octave spectrum. A larger number of averages and continuous recording can be used if the signal lasts sufficiently long. Additionally, the air pressure, air flow rate and the push force and grip force should also be recorded and kept as close as possible to the same levels during all the tests.

To maintain the same operating conditions between different operators or testings is not an easy task. Therefore, mounting the rivet hammer or the bucking bar on a test fixture to eliminate factors associated with the human operator is general in laboratory testing. Figure 9 shows a rivet hammer test fixture where the rivet hammer can be mounted in the middle of two supported tracks and is pushed by a double-acting air cylinder to press the hammer against the metal block. Different arrangements can be designed such as mounting the bucking bar on the opposite side on a similar track against the rivet hammer with or without a sheet of metal between them. The rivet hammer and the bucking bar 


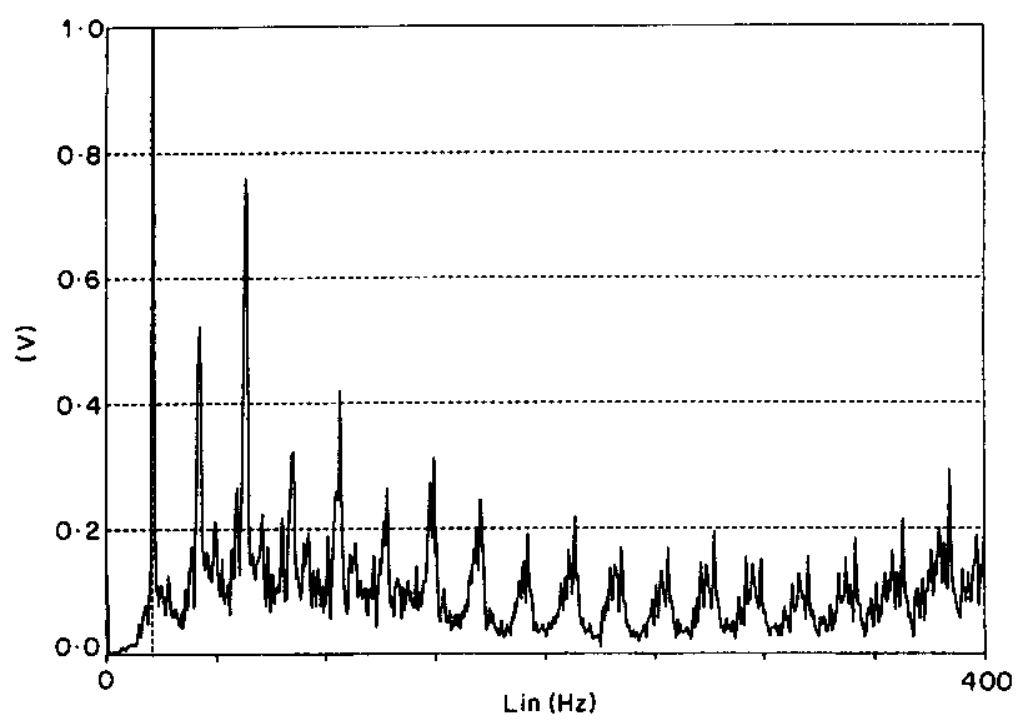

Figure 9. Frequency spectrum of a rivet hammer in the $400 \mathrm{~Hz}$ band.

are pressed against each other and the rivet hammer is usually triggered by a remote control switch.

The advantages of using a test fixture are: (1) that the duration can be much longer than with human operation and (2) the variations between different tests due to the push pressure, grip forces, operation time, and the alignment of the hammer can be much reduced therefore more consistent data are achieved.

The disadvantages of this method are: (1) since the hammer or the bucking bar is no longer held by human hand, the test condition is no longer considered to be representative of the actual field operating conditions and (2) only one direction of vibration can be measured.

The number of linear averages can be much greater such as 20 or 50 but within the time duration of the hammering. Consequently, continuous recording is used instead of single recording. Using a B \& K 2034 FFT analyser, the analyser will continue to feed the data

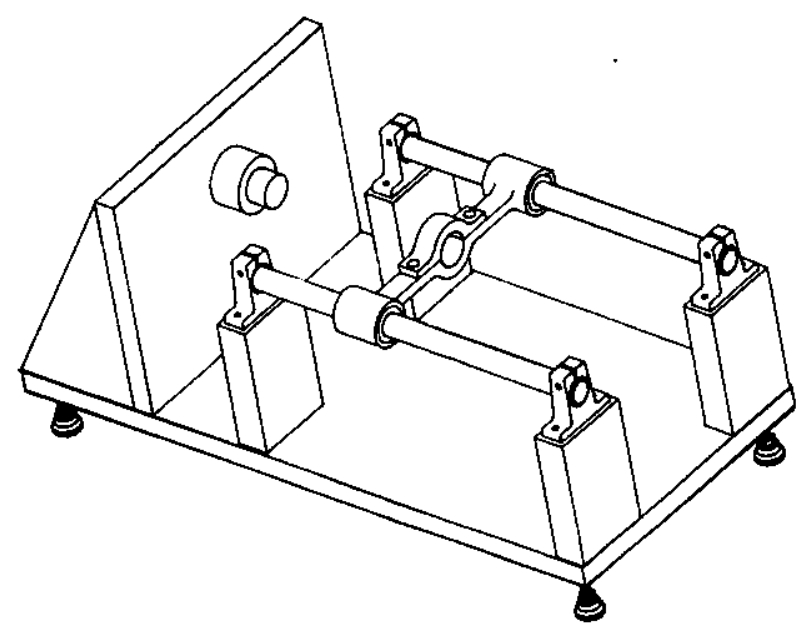

Figure 10. A test fixture for rivet hammer testing (courtesy of U.S. Industrial Tool \& Supply Company). 
into the screen even though the number of averages is complete, this will leave a partially filled time signal on the screen. One may want to push the stop button immediately after the number of averages is computed so that a complete time signal can be kept on the record.

If a real-time octave band DFA is used, the one-third octave spectrum can be obtained immediately from the testing. The operating time should be longer than $10 \mathrm{~s}$ in order to meet the requirements of the BT-product and to have high confidence levels at lower centre frequency bands.

To obtain the one-third octave frequency spectrum from a FFT analyser, a high

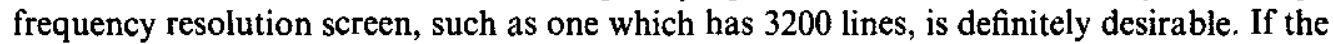
analyser has only 800 lines, alternatives are: (1) recording the data on a tape recorder as we suggested in the integration method and (2) using two identical FFT analysers simultaneously, one set at the $400 \mathrm{~Hz}$ frequency range and the other set at the $1.6 \mathrm{kHz}$ range. The input signal can then be branched into both analysers at the output of the charge amplifier. Obviously, the second alternative is only for the person who has two or more FFT analysers but no real time DFA analyser.

\section{CONCLUSIONS}

An intensive study on the measuring methods and analyses of transient multiple impulsive signals generated by pneumatic percussive rivet hammers and bucking bars has been completed. Because of the characteristics of the signals there is difficulty in generating the ISO 5349 standard required one-third octave frequency spectrum with high confidence levels in low frequency bands. These difficulties will exist if either a real time DFA or a FFT analyser are used in a single vibration measurement.

Two measuring methods that can overcome the measuring difficulties of percussive tools are presented in the paper. The advantages and disadvantages of each method are discussed in detail, additionally the relevant measurement and display set-ups of a real time DFA and FFT analyser are included.

Mounting the rivet hammer and the bucking bar in a test fixture can eliminate many noise factors such as the grip force and the push pressure of a human operator. This method is recommended for comparative testing in the laboratory for design modifications. For ergonomic studies, the testing tool is better held in a human hand, the use of a tape recorder and integrating multiple rivetings is then necessary.

If one's main interest is to generate the one-third octave frequency spectrum, a real time DFA with a tape recorder or a mounting fixture is the simplest arrangement. A real time DFA with a higher resolution frequency spectrum such as one-twelfth octave would be an advantage.

If a FFT analyser is used for the purpose of looking into the vibration harmonics, then a high resolution FFT analyser with a tape recorder or a mounting fixture is the best solution. A high resolution FFT analyser, i.e. 3200 frequency lines, can provide sufficient readings to generate the one-third octave spectrum required by the ISO standard. Low resolution FFT analysers, such as those with 400 or 800 frequency lines will have an accuracy problem in converting the FFT spectrum into a one-third octave spectrum. Alternatives are available, such as retrieving the data twice to a low resolution FFT analyser from a tape recorder or using two FFT analysers simultaneously.

It is important to understand the principles of vibration measurement and signal processing, but it is even more important to apply these principles to practical problems. This paper has linked an actual vibration measuring problem to the fundamental principles 
of digital signal processing such as sampling frequency, sampling time, and frequency resolution requirements.

\section{ACKNOWLEDGEMENT}

The research work presented in this paper is supported in part by a grant from the U.S. Industrial Tool and Supply Company, the authors are very grateful for the support provided by $\mathrm{Mr}$ C. Peterson, Operations Manager of the company. The authors also thank Mr J. A. Mathey, Mr G. Ebbit, and Mr K. Radwanski, B \& K Instruments, Inc. for their comments and suggestions.

\section{REFERENCES}

1. International ORganization for Standardization 1986 IOS 5349-1986(E). Mechanical vibration-guidelines for the measurement and the assessment of human exposure to hand-transmitted vibration.

2. M. FARKILA 1980 European Journal of Applied Physiology 43, 1-9. Vibration induced disease in the muscle force in lumberjacks.

3. W. Taylor, P. L. Pelmear and J. C. G. Pearson 1975 Vibration White Finger in Industry, pp. 1-13, London; Academic Press.

4. W. TAYLOR 1982 Journal of the Society for Occupational Medicine 32, 159-166. Vibration white finger in the workplace.

5. R. G. Radwin and T. J. ARMSTRong 1985 American Industrial Hygiene Association Journal 46, 211-219. Assessment of hand vibration exposure on an assembly line.

6. D. E. O'CONNOR and B. LindQuist 1990 Journal of Impact Measurement, 97-101. Method for measuring the vibration of impact pneumatic tools.

7. K. Engstrom and R. Dandanell 1986 Scandinavian Journal of Work and Environmental Health 12, 293-295. Exposure conditions and Raynaud's phenomenon among riveters in the aircraft industry.

8. R. B. RaNDall 1987 Frequency Analysis, Denmark: K. Larsen.

9. S. GADE and H. HeRLufsen $1987 B \& K$ Technical Review 3, 1-26. Use of weighting functions in DFT/FFT analysis. 